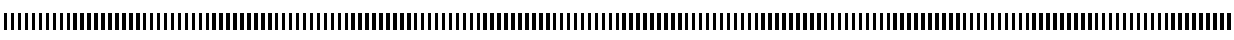

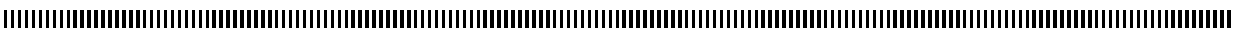

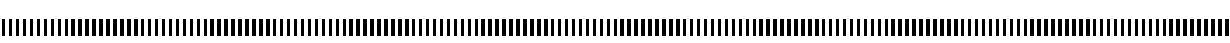

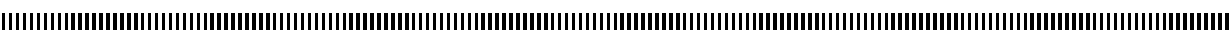

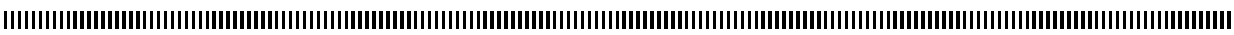

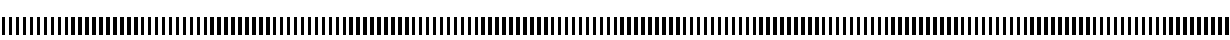

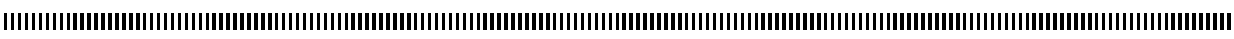

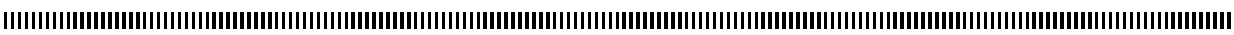

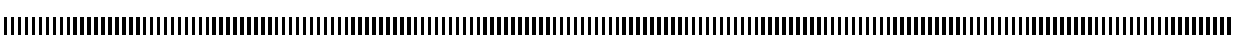

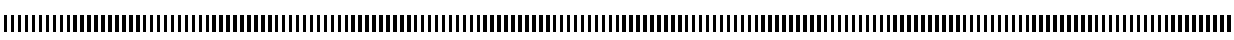

\title{
A Water Supply Optimization Problem for Plant Growth Based on GreenLab Model
}

\author{
Lin $\mathrm{Wu}^{12}$ - Philippe De Reffye ${ }^{3}$ - Bao-Gang $\mathrm{Hu}^{2}$ \\ - François-Xavier Le Dimet ${ }^{1}$ — Paul-Henry Cournède ${ }^{4}$ \\ 1 Université Joseph Fourier, Projet IDOPT, LMC-IMAG, Grenoble, France \\ \{Lin.Wu, Francois-Xavier.Ledimet\}@imag.fr \\ 2 Chinese Academy of Sciences, IA, LIAMA, 100080, Beijing, China \\ bubg@nlpria.ac.cn \\ 3 Projet DigiPlante, INRIA Rocquencourt, France \\ philippe.dereffye@inria.fr \\ 4 Laboratoire de Mathématiques Appliquées, Ecole Centrale Paris, France \\ cournede@ecp.fr
}

|

ABSTRACT. GreenLab is a structural-functional model for plant growth based on multidisciplinary knowledge. Its mathematical formalism allows dynamic simulation of plant growth and model analysis. A simplified soil water balance equation is introduced to illustrate the interactions and feedbacks between the plant functioning and water resources. A water supply optimization problem is then described and solved: the sunflower fruit weight is optimized with respect to different water supply strategies in a theoretical case. Intuitive searching method and genetic algorithms are used to solve this mixed integer nonlinear problem. The optimization results are analyzed and reveal possible agronomic applications.

RÉSUMÉ. GreenLab est un modèle structure-fonction de croissance des plantes. Son formalisme mathématique permet la simulation dynamique de la croissance et l'analyse du modèle. Dans cet article est introduit une équation bilan de l'eau dans le sol afin de décrire les interactions entre la croissance de la plante et les ressources en eau disponibles. Un problème d'optimisation des apports d'eau au cours de la croissance est présenté et résolu par la méthode de recherche intuitive et par les algorithmes génétiques : le poids du fruit de tournesol est maximisé en fonction de différentes stratégies d'apports d'eau, pour une quantité d'eau totale identique. Le formalisme présenté est intéressant en ce qu'il ouvre la voie à d'importantes applications en agronomie.

KEYWORDS : plant growth model, soil water balance, genetic algorithm, mixed integer nonlinear programming problem

MOTS-CLÉS : modèle de croissance de plantes, resources en eau, algoritmes génétiques, problème d'optimisation mixte

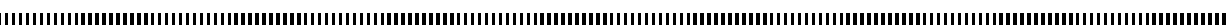

\section{Novembre 2005, Numéro spécial CARI'04}

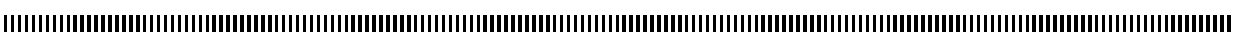

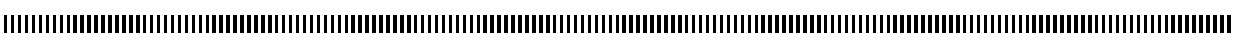

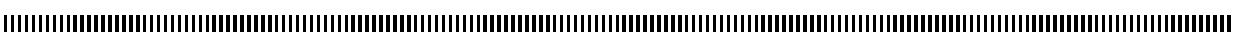
|

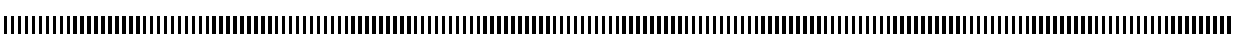

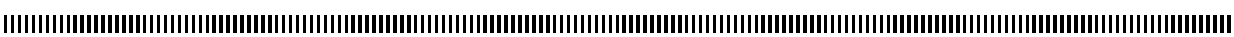

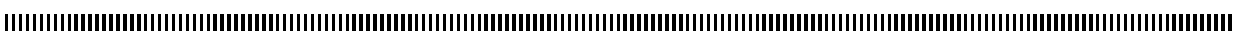
| 


\section{Introduction}

Plant growth models play an essential role in agronomy, botany and computer graphics. Different kinds of models, such as process-based models [6], geometric models [3] or functional-structural models [8], have been developed for special applications and they usually remain valid only for these. GreenLab model, see [2], tries to present a more general mathematical description of plant growth combining both physiology and architecture. It is based on multidisciplinary knowledge, including botany, eco-physiology, agronomy, mathematics and computer sciences. The development of GreenLab is a constant process of balancing the simplicity and complexity when choosing and adapting the biological and mathematical knowledge to form an efficient dynamical plant model useful for a wide variety of applications in agronomy and forestry [7].

In this paper, a simplified soil water balance equation is introduced to GreenLab model in order to take into account the interactions and feedbacks between the plant functioning and water resources. A water supply optimization problem is then formularized and solved with genetic algorithms, showing possible applications of GreenLab in optimization and control for agronomy.

\section{GreenLab model}

Like its predecessor AMAPhydro [1], GreenLab describes plant architecture at organ level. The evolution of the plant structure called organogenesis is periodical and we define the growth cycle (GC) as the thermal time necessary for each plant axis to develop a new growth unit (GU). Such a growth unit is composed of one or several elementary structure units that are a compound of associate organs (such as fruits and leaves) with an internode on stem. The duration of GC can vary from several days (cottons) to one year (temperate trees), but the sum of the daily temperatures needed to create a new GU of each GC is quite constant.

At every GC, the plant produces biomass by leaf photosynthesis. If we consider that every leaf undergoes the same microclimatic conditions, $Q(n)$ the biomass produced by the photosynthesis of all the leaves during GC $n$ can be formularized as an empirical nonlinear function $F$ of the environmental conditions $E(n)$, the number of leaves, their surface areas, and some hidden parameters $r 1, r 2 \ldots$ In GreenLab [1], we choose:

$$
Q(n)=F(S, E(n), r 1, r 2)=\sum_{a=1}^{n}\left(N_{L}(a, n) \cdot \frac{E(n)}{\frac{r_{1}}{S(a, n)}+r_{2}}\right)
$$

where $N_{L}(a, n)$ is the number of leaves $n$ of chronological age (CA) $a$, at GC $n$, (these leaves appeared at GC $n-a+1$, and $S(a, n)$ is their surface area. If the leaf thickness 
is constant, its surface area will be proportional to the biomass accumulated by the leaf. $r 1, r 2$ are parameters of $F$ to assess. $E(n)$ is the average biomass production potential depending on environmental factors, such as light, temperature and soil water content.

The biomass produced by photosynthesis is redistributed among all the organs according to their demands $d_{o}$ :

$$
d_{o}(j)=P_{o} \phi_{o}(j)
$$

which depends on organ CA $j$ and organ type $o(o=a, e, c, f, m$ and refers respectively to leaf, internode, layer, female flower and male flower). $P_{o}$ are the organ sink strengths and are model hidden parameters. $\phi_{o}$ are normalized distribution functions characterizing the evolution of the sink strengths from CA 1 to CA $t_{o}, t_{o}$ being the organ lifespan. Thus, the total biomass demand of the plant at GC $n$ is:

$$
D(n)=\sum_{o} \sum_{a=1}^{t_{o}} N_{o}(a, n) \cdot d_{o}(a)
$$

where $N_{o}(a, n)$ is the number of o-type organs of CA $a$ at GC $n$. This instantaneously leads to the calculation of the biomass increment $\Delta q_{o}(a, n)$ and total cumulated biomass $q_{o}(a, n)$ of any o-type organ of CA $a$ at current GC $n$ :

$$
\begin{aligned}
& \Delta q_{o}(a, n)=\frac{d_{o}(a, n)}{D(n)} \cdot Q(n-1) \\
& q_{o}(a, n)=\sum_{j=1}^{a} \Delta q_{o}(j, n-(a-j))=P_{o} \sum_{j=1}^{a} \frac{\phi(j) \cdot Q(n-(a-j)-1)}{D(n-(a-j))}
\end{aligned}
$$

\section{Mathematical description of the water supply problem}

\subsection{Plant growth interacting with water resources in soil}

Plants participate to soil water circulation by transpiration. Water is taken from soil by roots and flows through the plant hydraulic network up to the leaves, where water is transpired to provide necessary energy fluxes for photosynthesis. The water content in the superior soil layers, named soil moisture, is important for the study of bio-geophysical processes in agricultural or forestry ecosystems. Soil water balance is achieved when we simplify this complex soil-plant system by concentrating on plant transpiration, soil evapotranspiration, and water supply from both irrigation and precipitations.

Suppose $Q_{w}(t)$ is the water content in soil per surface unit. It can be considered as a potential. The loss of water by evapotranspiration is:

$$
d Q_{w}(t)=-c_{1} \cdot\left(Q_{w}(t)-Q_{w m n}\right) \cdot d t
$$


where $Q_{w m n}$ corresponds to the wilt point of soil water content beneath which the plant cannot extract water from soil. $c_{1}$ is an evapotranspiration coefficient. Likewise, if $U(t)$ is the water supply at $t$, the water gained by the soil is

$$
d Q_{w}(t)=c_{2}\left(Q_{w m x}-Q_{w}(t)\right) U(t) \cdot d t
$$

where $Q_{w m x}$ is the water field capacity above which the water flows away and $c_{2}$ is an absorption coefficient.

Thus the differential equation for the evolution of the soil water content is:

$$
d Q_{w}(t)=\left(-c_{1}\left(Q_{w}(t)-Q_{w m n}\right)+c_{2}\left(Q_{w m x}-Q_{w}(t)\right) \cdot U(t)\right) d t
$$

Figure 1 shows the fitting results of the calibrated soil moisture model (7) using measurements of the soil water content and rainfalls done in Ivory Coast.

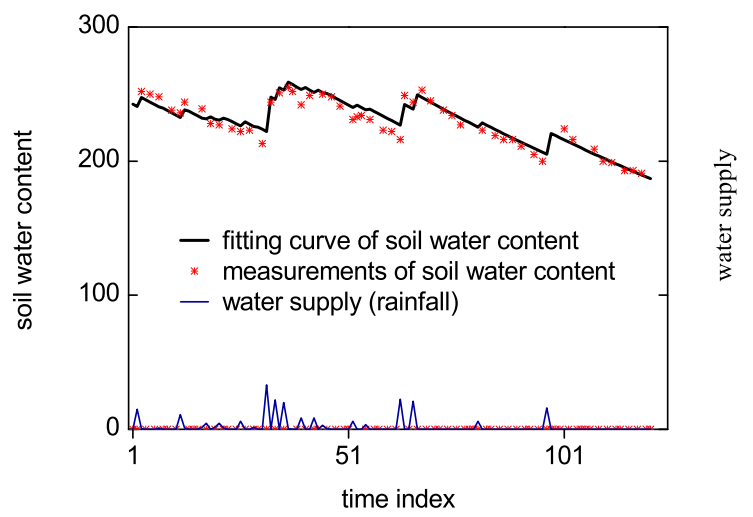

Figure 1. Fitting results of the calibrated soil moisture model

Considering plant transpiration, Equation (7) becomes:

$$
\frac{d Q_{w}(t)}{d t}=\underbrace{-c_{1}\left(Q_{w}(t)-Q_{w m n}\right)}_{\text {soil evapotranspiration }}+\underbrace{c_{2}\left(Q_{w m x}-Q_{w}(t)\right) \cdot U(t)}_{\text {water absorption }}-\underbrace{P T(t)}_{\text {plant transpiration }}
$$

where $P T(t)$ is the plant transpiration and is linearly proportional to plant biomass production calculated by equation (1). The discretized form of Equation (8) at GC scale is:

$$
Q_{w}(n+1)=\left(1-c_{1}-c_{2} \cdot U(n)\right) Q_{w}(n)+Q_{w m n} c_{1}+Q_{w m x} c_{2} \cdot U(n)-\rho \cdot Q(n)
$$


where $Q_{w}(n)$ is the soil water content at $\mathrm{GC} n, U(n)$ is the water supply during GC $n, Q(n)$ is the plant biomass production during $\mathrm{GC} n$ and $\rho$ is the ratio between plant transpiration and plant biomass production.

The biomass production potential $E(n)$ during GC $n$ is linearly proportional to the current soil water content, if we suppose that light and temperature conditions are optimal and the plant suffers no stress (for a complete formula, please refer to [10]):

$$
E(n)=K \cdot \frac{Q_{w}(n)-Q_{w m n}}{Q_{w m x}-Q_{w m n}}
$$

Equations (1), (4), (9) and (10) provide the whole mathematical formalism of the soilplant system that enables us to study the interactions between plant and water resources in soil. Note that all organ morphologic characteristics can be calculated by (4) and some additional allometric rules. They can be considered as observations of the soil-plant system. Once the observations are measured, hidden model parameters can be tracked back by inverse methods. In [2], it was shown that $E$ the calibration of some hidden parameters, such as sink strengths $P_{o}$ and hydraulic resistances $r_{1}, r_{2}$ remain stable. Therefore these parameters can be considered as internal endogenous factors. In [11], Zhan calibrates these parameters for several cultivated plants, such as maize, cotton, sunflower and tomato. In this preliminary study, we choose a 63 GC sunflower for the water supply optimization problem. The internal endogenous parameters are taken from the calibrated ones in [11], and the external environmental parameters, such as $c_{1}, c_{2}$, etc., are set to empirical values from previous studies. Agronomic experiments are difficult to design in order to validate the theoretical optimum. However, for some specific watering strategies (including the theoretical optimal one), it would be interesting to check the validity of the prediction.

\subsection{Formulation of plant water supply optimization problem}

In numerous cases, water reserves are limited because of drought or economic reasons. For a given quantity of water supply, the fruit yield will depend on the irrigation strategy during the plant growth.

In order to alleviate the calculation load, instead of optimizing the water supply at each GC, we try to find an optimal water distribution curve of the total water supply amount among a cluster of curves. We use parameterized beta function to generate a cluster of water supply curves. Given beta function parameters $a$ and $b$, and the total water supply amount WT, the water supply at GC $i, i \in[1, N]$, noted $U_{i}$, is:

$$
U_{i}=\frac{\mathrm{WT}}{S}\left(\frac{i-0.5}{N}\right)^{a-1}\left(1-\frac{i-0.5}{N}\right)^{b-1}
$$

where $S$ is a normalization factor of beta function: 


$$
S=\sum_{i=1}^{N}\left(\frac{i-0.5}{N}\right)^{a-1}\left(1-\frac{i-0.5}{N}\right)^{b-1}
$$

We introduce two variables date, the GC at which the water supply starts, and freq, the water supply frequency, to simulate a practical water supply strategy. The water supply is redistributed at GC $i$, with $i=j \times$ freq + date, $j \in \mathrm{N}$ and $1 \leq i \leq N$. For the other GC, water supply is set to zero. The upper in figure 2 shows the curve cluster of beta law when $a=k, b=2 \times k, k=1,2,3,5,10,20$, date $=1$, freq $=1$, and rhythmically water supply is shown in the lower of figure 2 where $a=5, b=10$, date $=1$, freq $=2$.

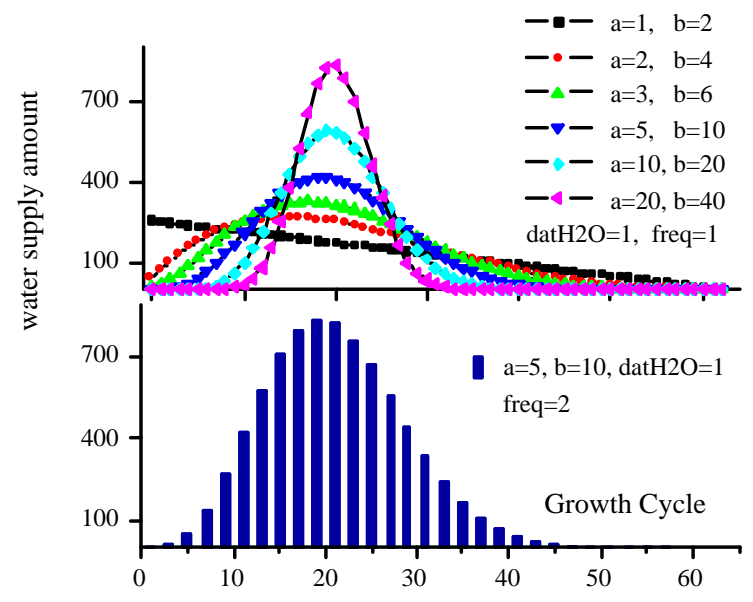

Figure 2. Curve cluster of beta law for continuous (upper) and rhythmical (lower) water supply, where $W T=8000, N=63$.

The water supply optimization problem is then formulated as a mixed integer nonlinear programming problem (MINLP) $\mathrm{P}$,

$$
\begin{array}{cc}
\max _{x \in \mathrm{R}^{4}} f(x) & \\
\text { subject to, } & 1 \leq x_{1}, x_{2} \leq 100 \\
& 1 \leq x_{3}, x_{4} \leq N
\end{array}
$$

where $x_{1}, x_{2}$ are the continuous beta law parameters $a, b$ and $x_{3}, x_{4}$ are the integer variables date and freq. The bounds for $x_{1}, x_{2}$ ensures a sufficient amount of distribution curves. $N$ is the sunflower total number of GC. 


\section{Approaches to the solution of problem (P)}

For comprehensive survey of algorithms and applications of MINLP, please see [4] and [9]. Unfortunately in problem $(\mathrm{P})$ the variable date and freq are fixed to be integers and there is no definition for real values. Therefore we can't define the relaxation problem by allowing date and freq to be real variables and by imposing boundary constraints in branch and bound algorithms. In addition, because of the nonconvexity of objective function (induced by thresholding, i.e. water field capacity $Q_{w m x}$ ), the introduction of master problem by linearizations incurs severe difficulties, i.e. the solution of the master problem does not have a valid bounding representation and the global optimum may be cut off in cutting plane methods.

Due to these reasons, we did not consider classic methods introduced in [4], but alternatively design an intuitive searching process of bug walk adapted to the special structure of the problem $(\mathrm{P})$. Then genetic algorithms are employed for the purpose of global optimum. Roughly speaking, the bug walk method terminates within one hour, but can only obtain local results, whereas using genetic algorithms we are more likely to find global solutions within several hours of calculation. The water supply optimization problem here is strongly non-convex (see [10]) and further theoretical investigations are necessary to prove that the optimum found is the global one. However, various numerical tests have reinforced this assumption. The significance of this paper is to define such water supply problems with better solutions.

\subsection{Solution of problem $(P)$ with bug search process}

We define problem (P1) for each fixed pair [date, freq] as follows

$$
\begin{array}{cc}
\max _{x \in \mathrm{R}^{2}} & f(x, \text { date, freq }) \\
\text { subject to, } & 1 \leq x_{1}, x_{2} \leq 100
\end{array}
$$

where $x_{1}, x_{2}$ are same as in problem (P). Problem (P1) is a typical bound-constrained problem and can be solved by standard optimization subroutines (in this paper MATLAB optimization subroutine fmincon is employed).

The bug search diagram and its pseudocode are shown in figure 3. The first step of the process is to generate center pair $O_{A}$ randomly and to solve problem (P1) at $O_{A}$. Then problem (P1) is solved at each pair around the center pair $O_{A}$ and we record the optimal fruit weight for each pair $O_{i}$, which is denoted OptimalValue $\left(O_{i}\right)$. Subsequently we choose clockwise the pair with better fruit weight as the new center pair. The searching continues until there is no pair around the center one that has better fruit weight. The optimization results are the final center pair value $[$ date, freq] and the optimal values of $a, b$ at the center pair. 
201 L. Wu, Ph. De Reffye, Bao-Gang Hu, F.-X. Le Dimet, P.-H. Cournède

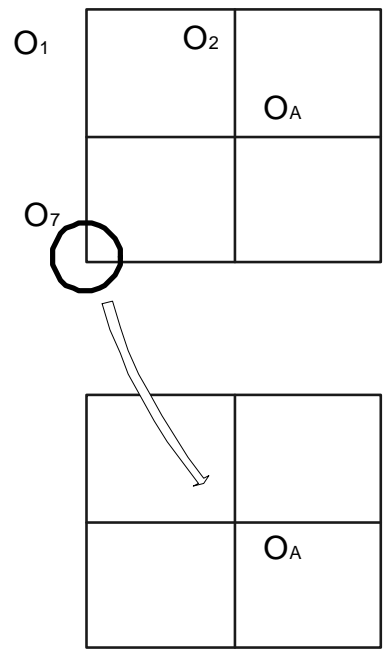

(a)

\author{
Initialize searching pair $O_{A}$ \\ While termination criteria not satisfied \\ Solve problem P1 at pair $O_{A}$ \\ Solve problems $\mathrm{P} 1$ at neighbor pairs \\ $O i, I=1 . .8$ \\ Find the maximum $O_{O}$ in the eight neighbor pairs \\ If OptimalValue $\left(O_{O}\right)==$ OptimalValue $\left(O_{A}\right)$ \\ Termination criteria satisfied \\ else \\ Moving searching pair to $O_{O}\left(O_{A}=O_{O}\right)$ \\ End If
}

End While

(b)

Figure 3. Diagram (a) and pseudocode (b) of search process for bug walk.

The bug search method is essentially a local algorithm. For the four initial pairs in table 1, beta law parameters $a$ and $b$ are all initialized as 3 and 12 respectively. The iteration results are shown in figure 4 . When freq is big enough, the shape of curve has little influence on the optimal results. The case (I) and case (II) have the same optimal $a$ and $b$ values. In contrast, it seems that frequency has significant influence on optimization results. Note that case (III) and (IV) have the same supply frequency but different initial supply date.

\begin{tabular}{|c||cc||cccc||c|}
\hline \multicolumn{1}{|c||}{} & \multicolumn{3}{c||}{ Initial pair } & \multicolumn{3}{c||}{ Optimal parameter values } & \multirow{2}{*}{ Optimal fruit } \\
\cline { 2 - 7 } & date & freq & $\mathrm{a}$ & $\mathrm{b}$ & date & freq & weight $(\mathrm{g})$ \\
\hline (I) & 18 & 61 & 3 & 12 & 32 & 63 & 112.42 \\
\hline (II) & 36 & 29 & 3 & 12 & 25 & 19 & 213.48 \\
\hline (III) & 4 & 12 & 1.3096 & 1.1532 & 2 & 2 & 1192.5 \\
\hline (IV) & 15 & 3 & 1.2063 & 1.1506 & 6 & 2 & 1170.5 \\
\hline
\end{tabular}

Table 1. Bug search method results with different initial pairs.

\section{Revue ARIMA}




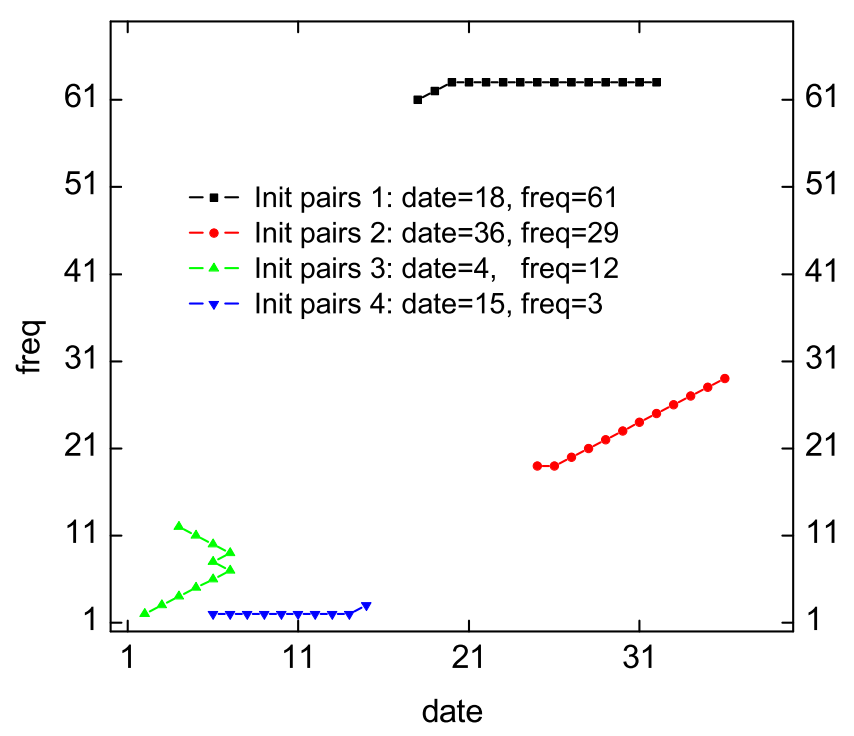

Figure 4. Iteration of bug search algorithm with different initial pairs in table 1.

\subsection{Solution of problem $(P)$ using genetic algorithms}

Genetic algorithms belong to a category of stochastic search techniques for optimization. A population of elements in the search space evolves generation after generation towards a better fitness, according to genetic-like rules. The GA used in this paper is a standard one [5]. The continuous parameters $a, b$, and integer parameters date, freq, are encoded as arrays of binary bits, named chromosomes. The fitness function is the final fruit weight obtained with the corresponding water supply strategy. To generate a new population, we first select chromosomes with a probability proportional to their fitness. Then, the pairwise selected chromosomes exchange parts of their chromosomes with a crossover probability $P c$. Finally, mutation is carried out by flitting bits randomly with a mutation probability $\mathrm{Pm}$.

In this paper we use binary coding and decoding schemes for the mixed integercontinuous variables $[a b$ date freq]. The bit number allocated to each variable is calculated as

$$
b i t=\left\lfloor\log 2\left(\frac{u b-l b}{A_{c}}+1\right)\right\rfloor
$$




\section{L. Wu, Ph. De Reffye, Bao-Gang Hu, F.-X. Le Dimet, P.-H. Cournède}

where $u b, l b$ are the up and low bound of variables, $A_{c}$ is the coding precision, i.e. for integer variables $A_{c}=1$, and for continuous variables $A_{c}=0.01$. Applying this formula with $u b, l b$ set as in (11) and with $N$ equal to 63 , the bits allocated for [a bdate freq] are [14 1466 respectively.

The code and decode formulae of chromosomes for the mixed integer-continuous variables are as follows: Coding formula for real variables is

$$
b v a l=\mathrm{D} 2 \mathrm{~B}\left((f v a l-l b) *\left(2^{b i t}-1\right) /(u b-l b)\right)
$$

where D2B is a function that transforms decimal code to binary code, fval is the decimal real variable value, bval is the chromosome binary code of fval. The decoding formula for real variables is

$$
f v a l=\mathrm{B} 2 \mathrm{D}(b v a l) * \frac{u b-l b}{2^{b i t}-1}+l b
$$

where B2D is a function that transforms binary code to decimal code. The coding formulae for integer variables are

$$
\begin{aligned}
& \text { len }=u b-l b+1 \\
& \text { count }=\left\lfloor\frac{2^{b i t}-1}{\text { len }\rfloor}\right. \\
& \text { bval }=\mathrm{D} 2 \mathrm{~B}((\text { ival }-l b)+\operatorname{randint}(1, \text { count }-1) * \text { len }))
\end{aligned}
$$

where function randint $\left(i_{1}, i_{2}\right)$ generates a random integer between $i_{1}$ and $i_{2}$. The decoding formula for integer variables is

$$
i v a l=\bmod (\mathrm{B} 2 \mathrm{D}(b v a l), u b-l b+1)+l b
$$

where $\bmod (i, D)$ is a function that calculates the remainder when integer $i$ is divided by integer $D$.

\begin{tabular}{|c||c||cccc||c|}
\hline \multirow{2}{*}{$\begin{array}{c}\text { Solution } \\
\text { Number }\end{array}$} & \multirow{2}{*}{$\begin{array}{c}\text { Appearing } \\
\text { Generation }\end{array}$} & \multicolumn{4}{|c||}{ Optimal parameter values } & Optimal fruit \\
\cline { 3 - 6 } & weight $(\mathrm{g})$ \\
\hline 1 & 110 & 1.3686 & 1.1934 & 1 & 2 & 1196.3 \\
\hline 2 & 92 & 1.3263 & 1.1632 & 1 & 2 & 1196.6 \\
\hline 3 & 108 & 1.3263 & 1.1571 & 1 & 2 & 1196.6 \\
\hline
\end{tabular}

Table 2. Optimization results of MINLP GA solver 
To solve the water supply optimization problem, we take a population size of 35 , $P m=0.05, P c=0.4$ and we stop the algorithm after 150 generations. The best chromosome is always kept for the next generation. The MINLP genetic algorithm solver was tried 3 times with randomly generated initial populations. The optimal parameter values, optimal fruit weight and the generation at which the best chromosome appears are listed in table 2. The optimal parameter values are stable for the 3 tries. The corresponding iterative processes are shown in figure 5.

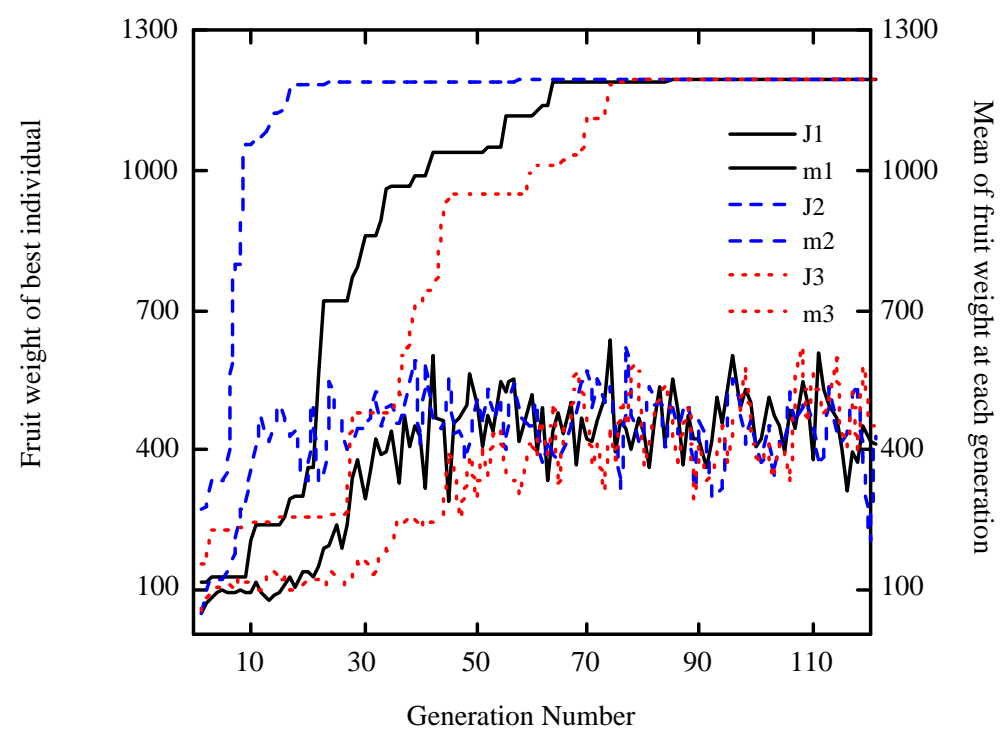

Figure 5. Iterative results of MINLP GA solver along generations, where J1-J3 are best fruit weight and m1-m3 the mean fruit weight along generations for solution 1-3 in table 2.

Different water supply strategies and their fruit accumulation are compared in figure 6. (a) is a linear water supply strategy, with freq $=1$, date $=1$; (b) is the optimal water supply strategy with freq $=1$, date $=1$; (c) is the optimal results of the problem $(\mathrm{P})$ with arbitrary freq and date values. 3D plant geometries of each strategy are also calculated and compared. The fruit weight $(\mathrm{J})$ and sunflower height $(\mathrm{H})$ are quite different for water distribution strategies (a), (b), (c). With the optimal strategy (c), the fruit is $18 \%$ heavier than with strategy (b) and the plant is $15 \%$ higher. It is interesting to note that with strategy (b), the fruit is $51 \%$ heavier than with strategy (a), but the plant is smaller. It is due to the abundance of water supply at early GC, favoring internode growth, and deficient water supply after the fruit appearance for strategy (a). 


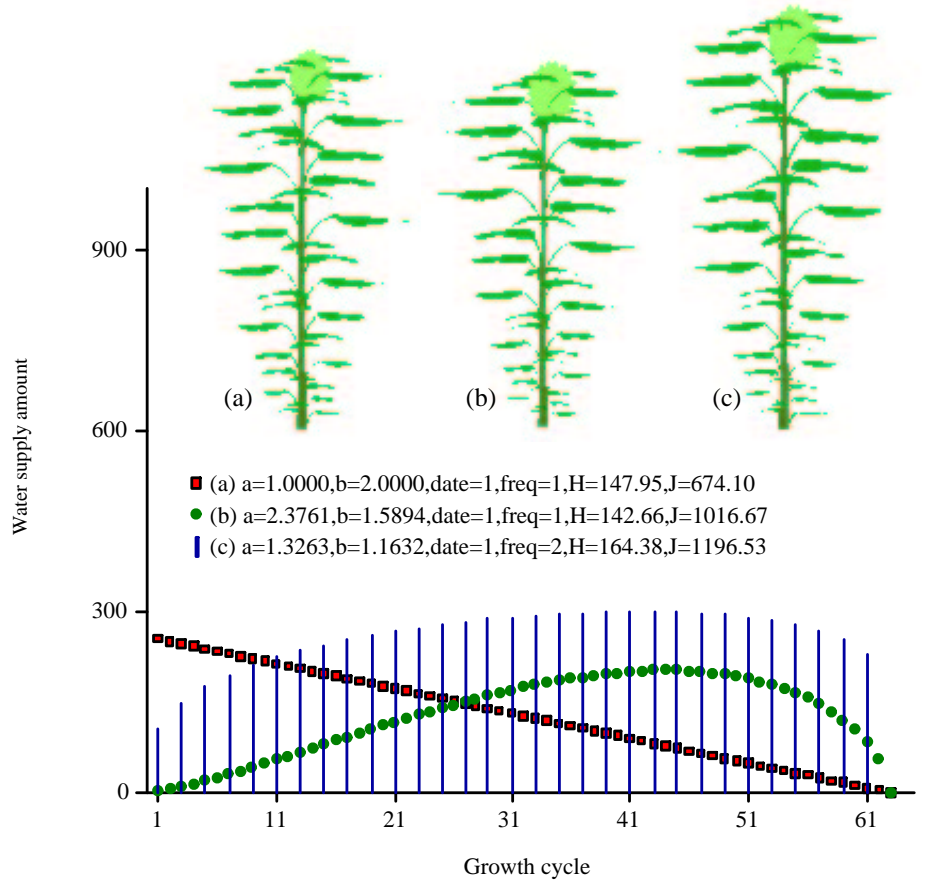

Figure 6. Comparison of different water supply strategies and the resulting fruit weight.

\section{Conclusion}

In this paper, we have extended GreenLab model in order to take into account available water resources in soil. It has been done by deriving a simplified water balance equation. The mathematical formalism introduced and the plant growth description by a dynamical system has allowed us to define a water supply optimization problem. It has been solved using genetic algorithms and the numerical results obtained in a theoretical case give the best water supply strategy in order to obtain a maximum fruit weight. The results are rather preliminary, since external parameters are set empirically, and their calibration and validation are needed. However, the problem solved is a very good example of the kind of applications in agronomy or forestry that we want to achieve. We are confident that the mathematical formalism introduced in the functional-structural model GreenLab should lead to this goal. 


\section{Acknowledgments}

This work is supported by LIAMA, China NSFC(\#60073007), China 863 Program (\#2002AA241221), and INRIA project IDOPT.

\section{References}

[1] De Reffye P., Blaise F., Chemouny S., Jaffuel S., Fourcaud T. , Houllier F., "Calibration of a hydraulic architecture-based growth model of cotton plants", Agronomie vol. 19, 1999 , p. $265-280$

[2] De Reffye P., Hu B.-G., "Relevant qualitative and quantitative choices for building an efficient dynamic plant growth model: GreenLab Case", In: Plant Growth Modeling and Applications: Proceedings - PMA03, Hu B.-G. and Jaeger M., (eds.), Tsinghua University Press and Springer, Beijing, China, 2003, pp. 87-107

[3] FrançON J., "Sur la modélisation informatique de 1 'architecture et du développement des végétaux", Colloque “l'arbre ”, Montpellier, 1990, p. 210-216

[4] Grossman I.E. , Kravanja Z., " Mixed-integer nonlinear programming: a survey of algorithms and applications ", in Large-scale optimization with applications Part II: Optimal design and control, A.R.Conn, L.T. Biegler, T.F. Coleman and F.N. Santosa (Eds), New York, Berlin, Springer, 1997

[5] Lin C.Y., HAJEla P., "Genetic algorithms in optimization problems with discrete and integer design variables”, Eng. Opt vol. 19, 1992, p. 309-327

[6] Marcelis L.F.M., Heuvelink E., GoudriaAn J. , "Modelling biomass production and yield of horticultural crops: a review", Scientia Horticulturae, 7, 1998, p. 83-111

[7] Nosenzo R., De Reffye, Ph., Blaise F., Le Dimet F.-X., "Principes de l'optimisation des modes de conduites culturales avec les modèles mathématiques de plantes", in Modélisation des agro-écosystémes et aide è la décision, CIRAD, Collection Repères, Montpellier, France, 2001, p. 145-172

[8] Sievänen R., Nikinmaa E., Nygren P., Ozier-Lafontaine H., Perttunen J., HAKUla H., "Components of a functional-structural tree model", Ann. For. Sci. vol. 57, 2000, p. 399-412

[9] Tawarmalani M., Sahinidis N.V., "Convexification and Global Optimization in Continuous and Mixed-Integer Nonlinear Programming, Theory, Algorithms, Software, and Applications ", Kluwer Academic Publishers, Dordrecht, Vol. 65 in "Nonconvex Optimization And Its Applications" series, 2002

[10] WU L., “ Variational Methods Applied to Plant Functional-Structural Dynamics: Parameter Identification, Control and Data Assimilation, " Thesis of Laboratoire de Modélisation et Calcul, Université Joseph Fourier, April 2005 
207 L. Wu, Ph. De Reffye, Bao-Gang Hu, F.-X. Le Dimet, P.-H. Cournède

[11] Zhan Z., Wang Y., De Reffye P., Wang Bingbing, Xiong Yanwen, "Architectural Modeling of Wheat Growth and Validation Study", ASAE Annual International Meeting, July, 2000

Revue ARIMA 